\title{
Níveis de vibração e choques em diferentes estradas durante o transporte de ovos férteis
}

\author{
Aérica C. Nazareno', Iran J. O. da Silva' ${ }^{1}$, Afrânio M. C. Vieira², \\ Frederico M. C. Vieira ${ }^{3}$ \& Késia O. S. Miranda ${ }^{1}$
}

\begin{abstract}
RESUMO
Os níveis de vibração e choques podem, devido ao transporte, afetar a qualidade dos ovos férteis. O objetivo desta pesquisa foi caracterizar e avaliar a influência das estradas sobre os níveis de vibração e choques no transporte dos ovos férteis. A pesquisa foi conduzida na empresa integradora avícola no estado de São Paulo, por meio do acompanhamento de três carregamentos em diferentes estações do ano (primavera, verão e outono). Um caminhão climatizado do tipo baú foi utilizado, com capacidade para 592 caixas de ovos, totalizando em média 90.840 ovos. As avaliações dos níveis de vibração ( $\mathrm{m} \mathrm{s}^{-2}$ ) e choques nas estradas de terra e asfalto foram registradas por meio de quatro acelerômetros da marca Hobo. Para o nível de vibração o delineamento utilizado foi inteiramente aleatorizado com esquema fatorial $3 \times 2 \times 4$. Para o número de choques o delineamento utilizado foi inteiramente aleatorizado com esquema fatorial $3 \times 2$. Os piores níveis de vibração e choques da carga ocorreram no sentido vertical. A estrada de asfalto ofereceu as piores condições para o transporte de ovos. As perdas no transporte ultrapassaram $1 \%$ e são atribuídas às trincas e quebra dos ovos.
\end{abstract}

Palavras-chave: avicultura, ambiência, transporte de ovos, operações pré-porteira

\section{Levels of vibration and shock on different roads during transportation of fertile eggs}

\begin{abstract}
The levels of vibration and shock due to transport can affect the quality of fertile eggs. The aim of this study was to characterize and to evaluate the influence of roads on the levels of vibration and shock in the transport of fertile eggs. The survey was conducted in the poultry integrator company in the State of São Paulo, by tracking shipments in three different seasons (spring, summer and autumn). An air-conditioned trunk was used, with a capacity of 592 boxes of eggs, totaling an average of 90,840 eggs. The evaluation of vibration levels $\left(\mathrm{m} \mathrm{s}^{-2}\right.$ ) and shocks on unpaved and asphalt roads were recorded by four accelerometers brand Hobo. For the vibration level, the experimental design was completely randomized in a $3 \times 2 \times 4$ factorial. For the number of shocks the experimental design was completely randomized with a factorial $3 \mathrm{x}$ 2. The worst levels of vibration and shock load occurred in the vertical direction. The asphalt road provided the worst conditions for the transport of eggs. The transport losses exceeded $1 \%$, which are attributed to cracks and breakage of eggs.
\end{abstract}

Key words: poultry production, animal environment, egg transport, prehatching operation

'Escola Superior de Agricultura "Luiz de Queiroz", Piracicaba, SP. Fone: (19) 3447-8563. E-mail: aericacn@yahoo.com.br; ijosilva@usp.br; kosilva@usp.br

${ }^{2}$ Universidade de Brasilia, Brasília, DF. Fone: (55) 6131-0767. E-mail: afranio.unb@gmail.com

${ }^{3}$ Universidade Tecnológica Federal do Paraná, Dois Vizinhos, PR. Fone: (46) 3536-8417. E-mail: fredvzoo@gmail.com 


\section{INTRODUÇÃO}

Os tipos de estrada (terra e asfalto) podem exercer sérios impactos na carga, o que deve ser considerado no planejamento de um transporte de ovos férteis. No caso de estradas de terra a irregularidade das pistas oferece outra influência nos ovos, embora pouco conhecida, que é a vibração da carga (Nazareno, 2012). Dependendo das condições das vias de transporte a qualidade dos ovos férteis pode ser comprometida. A proporção de choques e vibrações aos quais a carga estará sendo submetida poderá afetar a qualidade do produto final (ovo fértil) e favorecer o aumento das perdas atribuídas às trincas e quebras (Mertens et al., 2005; Wang \& Jiang, 2005; Bain et al., 2006; Mertens et al., 2006; Bamelis et al., 2008; Deng et al., 2010; Nazareno, 2012). Então, quanto pior a condição da estrada maior será o tempo gasto na viagem; logo, consequentemente maior será também o tempo em que os ovos férteis ficarão expostos às condições adversas.

A vibração pode ser definida como um movimento de oscilações de um corpo em torno de sua posição de equilíbrio (Lida, 2005). Esta é definida por três variáveis, que são: a frequência, que é uma grandeza física ondulatória que indica o número de ocorrências de um evento (ciclos, voltas, oscilações, etc.) em determinado intervalo de tempo; a intensidade do deslocamento ( $\mathrm{cm}$ ou $\mathrm{mm}$ ) ou aceleração máxima sofrida no corpo, medido em gravidade $\left(1 \mathrm{~g}=9,81 \mathrm{~m} \mathrm{~s}^{-2}\right)$ e a direção do movimento, definida por três eixos triorthogonal: $\mathrm{X}$ sentido vertical "do piso à parte superior", Y sentido lateral "lado esquerdo para o direito" e $\mathrm{Z}$ sentido horizontal "da frente para a traseira" (Lida, 2005; Walber \& Tamagna, 2010).

A salientar que os níveis vibratórios de um veículo de transporte (caminhão e/ou ônibus) podem variar com o tipo de suspensão, número de eixos, calibragem de pneus, tipo de amortecedores, velocidade (constante, aceleração ou frenagem), tipo e peso da carga, qualidade das estradas e rodovias etc. (Pierce et al., 1992; Franchini, 2007; Idah et al., 2009; Walber \& Tamagna, 2010).

Alguns autores discutiram os efeitos da vibração em ovos. Lida (2005) comenta que a vibração é um fator que influencia o transporte de ovos pois, dependendo da estrada e do caminhão, ocorrerão movimentos que podem ser regulares ou irregulares, levando o caminhão a trepidar. Portanto, essa trepidação pode agredir a casca do ovo provocando danos mecânicos, como trinca na casca, agitamento do material interno do ovo, danificação do albúmen e da qualidade da gema causando, assim, perda dos ovos férteis (Thompson et al., 1986; Altuntaş \& Şekeroğlu, 2008; Deng et al., 2010).

Os efeitos da vibração no transporte de ovos também foram estudados por Berardinelli et al. (2003), que avaliaram a influência dos níveis de vibração em caminhões de ovos sobre os parâmetros que descrevem a qualidade dos ovos: unidade Haugh, força da membrana vitelínica e altura da camada de ar. Os autores observaram que as maiores acelerações ocorreram na faixa de frequência de $5-20 \mathrm{~Hz}$ condicionando, assim, uma diminuição de $28 \%$ na unidade Haugh, demonstrando que esses ovos podem parecer cerca de 10 dias mais velhos em relação aos ovos que não sofreram nenhum nível de vibração; houve uma redução de $18 \%$ da força da membrana vitelínica e aumento na altura da camada de ar do ovo.
O impacto econômico das perdas decorrentes das operações pré-porteira, em 2011, pode ser avaliado na seguinte condição: o Brasil produziu 31,5 bilhões de ovos que correspondem aos ovos férteis, incubados e consumo humano (Turra, 2012). Atualmente, o custo médio de produção por ovo é em torno de R $\$ 0,60$ entre os dez maiores estados produtores do Brasil. Conforme esses dados e caso se considere que durante o transporte ocorrem perdas de $1 \%$ atribuídas a trincas e quebras que foram provocadas por transportes inadequados e/ou operações executadas de maneira errada nesta etapa pré-porteira, podem ter gerado um prejuízo anual na indústria avícola nacional, na ordem de $\mathrm{R} \$ 18,9$ milhões (Nazareno, 2012).

Partindo do princípio de que a avicultura precisa rever alguns de seus processos de manejo, especialmente com relação às etapas pré-porteira, visando à redução de perdas, a presente pesquisa teve como objetivo principal caracterizar e avaliar a influência das estradas sobre os níveis de vibração e choques no transporte dos ovos férteis.

\section{Material e Métodos}

A pesquisa foi conduzida nas instalações do matrizeiro e do incubatório avícola de uma empresa integradora localizado no município de Mogi Mirim, no Estado de São Paulo, apresentando latitude de $22^{\circ} 25^{\prime} 55^{\prime \prime} \mathrm{S}$, longitude de $46^{\circ} 57^{\prime} 28^{\prime \prime} \mathrm{W}$ e altitude de $632 \mathrm{~m}$. Os períodos experimentais foram de novembro a dezembro de 2010 e de fevereiro a abril de 2011, durante a primavera, verão e outono, acompanhando três carregamentos. Foi utilizado um caminhão de transporte do tipo baú, modelo Constellation 26-370 $6 \times 4$ tractor da Volkswagen, apresentando as dimensões de 7,5 $\mathrm{m}$ de comprimento, 2,6 $\mathrm{m}$ de largura e 2,6 $\mathrm{m}$ de altura, com três eixos. A calibragem dos pneus é feita com 100 libras, o tipo de suspensão é com molas parabólicas e amortecedores hidráulicos de dupla ação. A capacidade de carregamento do caminhão era de aproximadamente 592 caixas de ovos empilhadas e distribuídas na área da carroceria. A carga total média dos carregamentos foi de 90.480 ovos.

As caixas de transporte de ovos possuem dimensões externas de $63 \mathrm{~cm}$ de comprimento, $32,5 \mathrm{~cm}$ de largura e $30 \mathrm{~cm}$ de altura e espessura $3 \mathrm{~mm}$, com abertura nas laterais de $3,2 \times 1,1 \mathrm{~cm} \mathrm{e}$ no fundo de $2 \times 2 \mathrm{~cm}$. Elas suportam 240 ovos que equivalem a 8 bandejas de plástico com as dimensões de 30 x $30 \mathrm{~cm}$ com capacidade de 30 ovos.

Durante o transporte foi avaliado o nível de vibração $\left(\mathrm{m} \mathrm{s}^{-2}\right)$ das quatro pilhas de caixas de ovos, que estavam localizadas nas quatro extremidades (frente direita e esquerda, traseira direita e esquerda) da carroceria do caminhão, registrado por meio de quatro acelerômetros triaxiais e data loggers da marca Hobo Pendant G Acceleration data logger, com faixa de medição de $\pm 3 \mathrm{~g}$ ou $29,4 \mathrm{~m} \mathrm{~s}^{-2}$, parafusado na parte interna da primeira caixa da pilha e no interior das mesmas, segundo os autores Berardinelli et al. (2003), conforme apresentado na Figura 1.

A medição foi registrada por meio da aceleração do caminhão em intervalos de um segundo com a frequência de $1 \mathrm{~Hz}$ constante. Durante a viagem o acelerômetro estabelece uma frequência fixa entre 1-100 Hz, conforme o intervalo de medição requisitado. $\mathrm{O}$ registro de dados se iniciava a partir do 

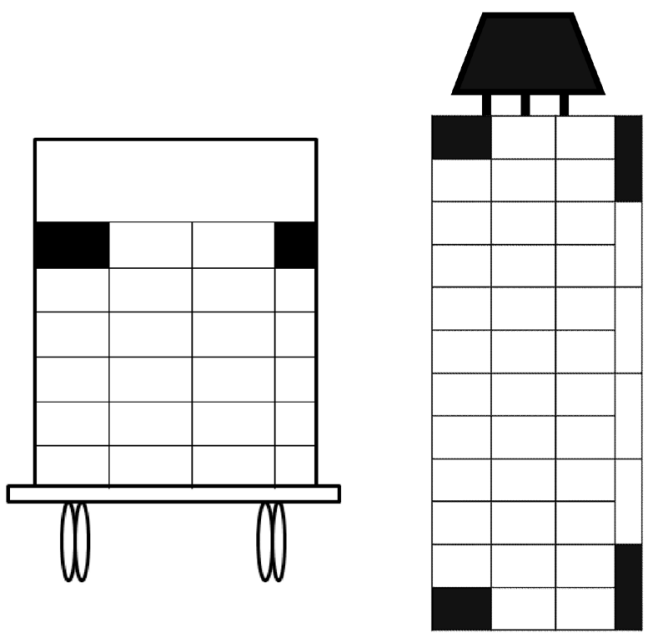

Figura 1. Distribuição fixa das posições das caixas instrumentadas pelos acelerômetros (caixas pretas) ao longo da carga

fechamento das portas do caminhão, quando foi quantificado seu tempo de permanência nas diferentes estradas. A viagem foi de $6 \mathrm{~km}$ em estrada de terra e $54 \mathrm{~km}$ em estrada asfaltada. Diante disto foi possível analisar a totalidade do trecho da estrada de terra e asfalto, avaliando a presença de choques mecânicos nas caixas analisadas.

Com os dados coletados da aceleração média (RMS) para cada um dos três eixos, pode-se estimar a aceleração geral (RSS) conforme as Eqs. 1 e 2 sugeridas por Randall et al. (1993; 1997).

$$
\mathrm{RMS}=\left(\frac{\sum \mathrm{a}_{\mathrm{i}}^{2}}{\mathrm{~N}}\right)^{\frac{1}{2}}
$$

em que:

$a_{i}$ - valor observado no eixo $(x, y$ ou $z)$ no tempo i $(i=$ $1,2, \ldots, \mathrm{N})$

$\mathrm{N}$ - número de observações no eixo (x, y ou z)

$$
\mathrm{RSS}=\left(\mathrm{RMS}_{\mathrm{x}}^{2}+\mathrm{RMS}_{\mathrm{y}}^{2}+\mathrm{RMS}_{\mathrm{z}}^{2}\right)^{\frac{1}{2}}
$$

em que:

x - vibração vertical do piso à parte superior

y - vibração lateral da direita à esquerda

z - vibração horizontal da frente ao fundo

A localização dos acelerômetros na carga do caminhão foi realizada de acordo com a metodologia dos autores Berardinelli et al. (2003).

Para a avaliação das perdas quanti-qualitativas durante $o$ transporte de ovos férteis foi contabilizado o número de ovos trincados e quebrados de toda a carga do caminhão.

O parâmetro ovos trincados e quebrados foi considerado fator de seleção ao processo, adotando-se o mesmo como variável resposta. A contabilização foi realizada conforme a rotina da empresa em que os funcionários selecionaram cada ovo, individualmente, pelo método visual. São critérios de seleção os ovos com trincas e quebras na casca, entre outros.
Na avaliação do nível de vibração da carroceria do caminhão adotou-se o delineamento experimental com estrutura de parcela do tipo inteiramente aleatorizada e, como estrutura de tratamento, o esquema fatorial de $3 \times 2 \times 4$, que representavam as três viagens, dois tipos de estrada (terra e asfalto) e quatro acelerômetros. A análise estatística utilizada foi o modelo linear normal assumindo, como variáveis respostas, as RMS X, RMS Y, RMS Z e RSS.

De acordo com as pesquisas de Randall et al. (1993; 1997), o número de choques de uma carga é representado pelos maiores valores das vibrações $\left(\mathrm{m} \mathrm{s}^{-2}\right)$ e/ou picos das vibrações ocorridos durante a viagem, seguidos de uma redução (frenagem) brusca na aceleração. Neste trabalho a proposta para avaliação do número de choques foi contabilizada por meio da utilização do bloxplot, que utiliza como referência o valor 1,5 de distância interquartilica, que seria a diferença entre o terceiro e primeiro quartil (Nazareno, 2012). O delineamento experimental utilizado foi a estrutura de parcela do tipo inteiramente aleatorizada e, como estrutura de tratamento, o esquema fatorial de $3 \times 2$, que representavam as três viagens e dois tipos de estrada (terra e asfalto). A análise estatística utilizada foi o modelo linear generalizado com distribuição binomial negativa e a função de ligação foi a logarítmica.

Todas as análises estatísticas foram realizadas por meio do software estatístico SAS (SAS, 2010).

\section{Resultados e Discussão}

Nas análises dos níveis de vibração constatou-se que não existe diferença estatística entre as viagens, estradas e posição dos acelerômetros para a RMS X (vibração vertical) e RMS Y (vibração lateral da direita à esquerda). Porém foi possível detectar diferença estatística entre as estradas de terra e asfalto para a RMS Z (vibração da carga no sentido da frente ao fundo) e RSS (aceleração geral). O maior valor médio do nível de vibração da RMS Z foi verificado na estrada de asfalto com 1,90 $\mathrm{m} \mathrm{s}^{-2} \mathrm{e}$ a menor média foi observada na estrada de terra com 1,2 $\mathrm{m} \mathrm{s}^{-2}$. Com relação à RSS (aceleração geral), observou-se que a estrada de asfalto obteve o maior valor médio de $13,1 \mathrm{~m} \mathrm{~s}^{-2} \mathrm{e}$ a menor média foi detectada na estrada de terra com 10,4 $\mathrm{m} \mathrm{s}^{-2}$.

Ao contrário do que se previa, a estrada de asfalto exerce uma vibração maior na carga de ovos que a estrada de terra $\left(\mathrm{RSS}_{\text {asfalto }}>\mathrm{RSS}_{\text {terra }}\right)$. Isto ocorre porque, em geral, o motorista dirige com maior cuidado na estrada de terra tendo em vista que esta apresenta, comumente, grandes irregularidades na pista, demonstrando que durante o percurso na estrada de asfalto o caminhão estava, na maior parte das vezes, com velocidade superior em relação à estrada de terra, quando só então foi possível observar um número maior de frenagem, resultando no RMS Z maior. Esta ação fez com que a carroceria do caminhão trepidasse mais intensamente causando maior vibração na horizontal, ou seja, deslocamento da carga no sentido da frente ao fundo e na aceleração geral.

Sabe-se que o nível de vibração no transporte de ovos vai depender da carga, do tipo de embalagem, da qualidade da estrada, velocidade (constante, aceleração ou frenagem), da distância, do tipo de suspensão e do número de eixos do caminhão, da calibragem de pneus e do tipo de amortecedores 
(Berardinelli et al., 2003; Franchini, 2007; Walber \& Tamagna, 2010).

Ao observar a Figura 2 verifica-se que os maiores valores médios de níveis de vibração ocorreram na estrada de asfalto, para todos os deslocamentos das cargas estudadas.

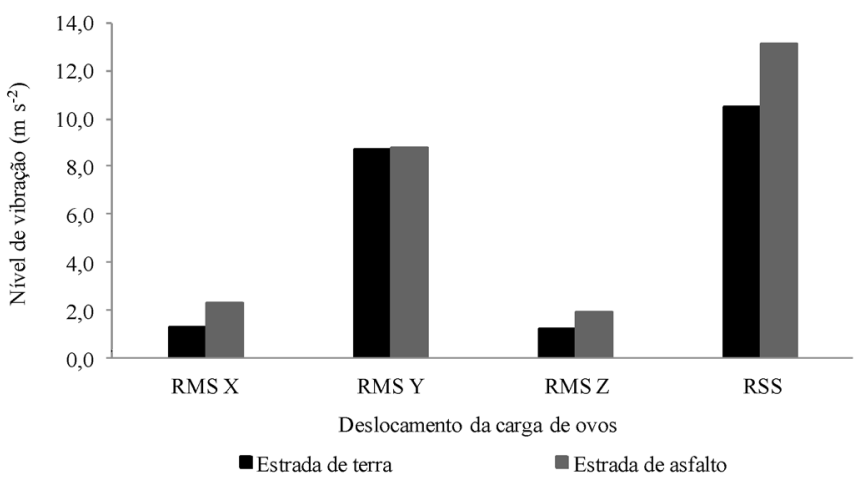

Figura 2. Valores médios dos níveis de vibração (m $\mathrm{s}^{-2}$ ) para os diferentes tipos de estrada (terra e asfalto), com os seus respectivos deslocamento da carga de ovos férteis e a faixa ideal do nível de vibração $(0,315$ $\mathrm{m} \mathrm{s}^{-2}$ ) para humano estabelecido pela ISO 2631-1

O nível de vibração pode influenciar, de maneira direta, as perdas pré-porteira no transporte de ovos férteis. Muitas pesquisas foram realizadas ao longo dos últimos anos com o objetivo de conhecer o comportamento do corpo humano quando exposto à vibração mecânica porém existem poucas pesquisas relacionadas ao transporte de ovos. A Norma ISO 2631-1 (1997) e os autores Walber \& Tamagna (2010) classificam como níveis de vibração de desconforto para humanos os valores maiores de $0,315 \mathrm{~m} \mathrm{~s}^{-2}$.

Portanto, ao comparar esse valor proposto pela ISO 26311 (1997) com os níveis de vibração no transporte de ovos, observa-se que as duas estradas apresentaram valores médios muito maiores que o recomendado. A estrada de terra apresentou maior tendência de valores médios próximos do ideal para todos os deslocamentos das cargas de ovos.

Ao avaliar o efeito do tipo de caminhão e suspensão sobre o nível de vibração em rodovias e estrada de terra, no transporte de frangos de corte, Randall et al. (1993) observaram que um caminhão de 14,8 toneladas, com suspensão a ar e nível de vibração de 0,18 a $0,42 \mathrm{~m} \mathrm{~s}^{-2}$, apresentou pouco conforto para as aves.

Os altos níveis de vibração podem aumentar a chance do aparecimento de trincas e quebras além de ocasionar o agitamento dos elementos constituintes do ovo (Mertens et al., 2005; 2006; Bain et al., 2006). Durante o trajeto do caminhão também existirá uma força que atuará nos ovos fazendo com que esses se agitem dentro das bandejas de plástico de forma contínua, podendo causar danos na casca do ovo. Randall et al. $(1993 ; 1997)$ e Zhan et al. (2009) ao avaliar o transporte de frangos de corte observaram que o animal dentro da caixa sente a vibração em virtude dos efeitos da aceleração e frenagem do caminhão, a qual é inércia, resultante que faz o animal mudar sua posição provocando estresse quando a aceleração é fraca ou forte, sendo a aceleração forte denominada choque (Figura 2).
Ao avaliar o transporte de ovos os autores Berardinelli et al. (2003) observaram que, além das perdas em virtude das trincas e rachaduras nos ovos férteis durante o transporte, o outro fator de perda seria o agitamento do material interno do ovo o qual pode danificar os elementos internos, tais como: albúmen, gema, casca, unidade Haugh, força da membrana vitelínica e altura da camada de ar. Portanto, o agitamento dos elementos internos do ovo pode ocasionar morte e má-formação embrionária além de interferir na eclodibilidade dos ovos férteis. Esses autores também observaram que os altos níveis de vibração interferiram na qualidade dos ovos de forma que esses apresentavam aparência de mais velhos.

Com relação à quantidade de choques aos quais a carga de ovos férteis estava sendo submetida, foi possível constatar que não houve diferença significativa para estradas (terra e asfalto) e viagens, para choques na vertical (piso à parte superior), ou seja, RMS X (Tabela 1). A respeito da RMS Y (vibração lateral da direita à esquerda) observou-se que o algoritmo de estimação dos parâmetros do modelo estatístico não convergiu para os valores confiáveis e o resultado da análise foi inconclusivo.

Tabela 1. Valores médios de choques na vertical representados pela variável RMS X e RMS Z em relação às viagens

\begin{tabular}{ccc}
\hline Viagens & RMS X & RMS Z \\
1 & $272,50 \mathrm{~A}$ & $497,71 \mathrm{~A}$ \\
2 & $415,70 \mathrm{~A}$ & $365,55 \mathrm{~A}$ \\
3 & $1439,70 \mathrm{~A}$ & $126,80 \mathrm{~B}$ \\
\hline Teste Qui-quadrado & $3,11 \mathrm{NS}$ & $12,48^{*}$ \\
\hline
\end{tabular}

Médias com letras diferentes minúsculas diferem entre si a nível de 0,05 de probabilidade pelo teste do Qui-quadrado. NS - não significativo, * - significativo a 0,05 $(\mathrm{P}<0,05)$

Para variável RMS Z (choques na vertical) detectou-se diferença estatística para as viagens e os dois tipos de estrada (terra e asfalto), $\operatorname{com} P=0,0019$. As viagens $1 \mathrm{e} 2$ não diferiram estatisticamente entre si porém diferiram da viagem 3. O maior valor médio da quantidade de choques ocorreu na viagem 1 com 497,71 e a menor média na viagem 3 , com 126,80 choques (Tabela 1).

A quantidade de choques que uma carga recebe está diretamente direcionada com o tipo de rodovia, veículo de transporte, número de ultrapassagens, frenagens e aumento da velocidade durante o percurso do caminhão etc. Muitas vezes a pressa de atender a logística de transporte da empresa pode levar a ocorrer um aumento ou diminuição no número de choques que a carga de ovos venha sofrer.

Com relação ao número de choques da variável RMS Z (choques na vertical) e aos dois tipos de estrada, foi possível observar que houve diferença estatística $(P=0,0019)$ entre a estrada de terra e de asfalto. Verificou-se que a estrada de asfalto apresentou a maior média de choques com 433,92 e a menor média foi encontrada na estrada de terra com 186,76 . O número de choques apresentou o mesmo comportamento do nível de vibração em que a estrada de asfalto foi a que apresentou a pior condição de transporte de ovos.

Essa tendência pode ser atribuída ao maior cuidado que o motorista teve ao dirigir no percurso de estrada de terra em decorrência da grande presença de irregularidades e buracos na pista. Deve-se considerar que o tipo e a qualidade da estrada, 
Tabela 2. Relação da carga total, número e porcentagem de ovos (trincados e quebrados) oriundos das três viagens

\begin{tabular}{cccccc}
\hline Viagens & $\begin{array}{c}\text { Carga total de ovos } \\
\text { no caminhão }\end{array}$ & $\begin{array}{c}\text { Número de ovos } \\
\text { trincados }\end{array}$ & $\begin{array}{c}\text { Ovos trincados } \\
\mathbf{( \% )}\end{array}$ & $\begin{array}{c}\text { Número de ovos } \\
\text { quebrados }\end{array}$ & $\begin{array}{c}\text { Ovos quebrados } \\
\text { (\%) }\end{array}$ \\
1 & 72.480 & 690 & 0,95 & 340 & 0,46 \\
2 & 112.080 & 994 & 0,88 & 240 & 145 \\
3 & 86.644 & 440 & 0,51 & 0,22 & 241,67 \\
\hline Média & $90.401,33$ & 708 & 0,78 & \\
\hline
\end{tabular}

a velocidade e o tipo do caminhão, influenciam diretamente (aumento ou diminuição) na quantidade de choques que a carga poderá sofrer (Randall et al., 1993; 1997; Lida, 2005).

De acordo com a Figura 3 foi possível observar que os maiores números de choques sofridos pela carga de ovos foram verificados na estrada de asfalto, para todos os deslocamentos da carga. Na literatura não foi possível encontrar o valor ideal para o número de choques que uma carga pode sofrer durante o transporte mas os autores Randall et al. (1993) comentaram que, quanto menor for o número de choques na carga transportada melhor será a condição de transporte.

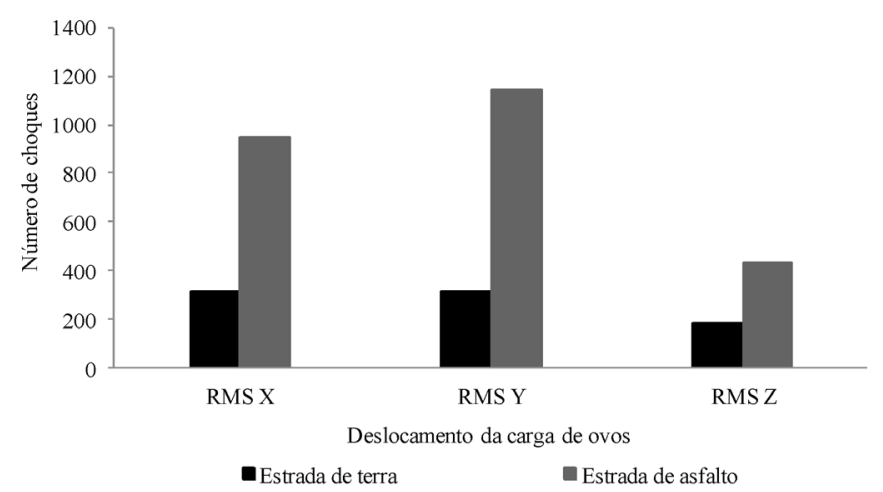

Figura 3. Valores médios do número de choques para os diferentes tipos de estrada (terra e asfalto), com seus respectivos deslocamento da carga de ovos férteis

Desta forma, pode-se dizer que o aparecimento das trincas e rachaduras nos ovos durante o transporte da carga pode ser atribuído ao maior número de choques e níveis de vibrações obtidos na estrada de asfalto, o que acarretou as piores condições de transporte e o que pode ter contribuído para o aumento das perdas ao longo da etapa de transporte.

Em função desta abordagem foi possível observar que existe uma carência em pesquisas que enfoquem os efeitos das diferentes estradas nos níveis de vibração e choques, que ocorrem no transporte, e seus reflexos na qualidade final dos ovos.

Na Tabela 2 se encontram a carga total, o número e a porcentagem de ovos que foram danificados (trincados e quebrados) durante as três viagens. Com essas variáveis podese verificar a influência do nível de vibração e dos choques na qualidade final e na integridade física dos ovos férteis.

Observa-se que a maior porcentagem de ovos férteis trincados ocorreu na viagem 1 , com $0,95 \%$. Com relação ao número e à porcentagem de ovos quebrados, os maiores valores ocorreram na viagem 1 ( 340 ovos e $0,46 \%)$. Esses resultados podem ser justificados pelo fato de que durante a viagem 1 houve o maior nível de vibração e choque na RMS Z (Tabela 1) comparados com as demais viagens.
Então, fazendo um somatório das perdas de ovos durante o transporte, verifica-se que elas ultrapassam $1 \%$ da carga, as quais são atribuídas ao alto nível de vibração e choques que, por consequência, causaram um aumento no número de trincas e quebras. Sendo assim, foi possível detectar que para uma carga total de 271.204 ovos férteis as perdas atribuídas ao transporte foram de 2.124 ovos trincados e 725 ovos quebrados, representando uma perda de $1,05 \%$ durante as três viagens. Entretanto, foi possível verificar, durante os três transportes de ovos férteis, que a empresa deixou de ganhar R $\$ 2279,20$ com a incubação desses ovos considerando o custo do ovo fértil de $\mathrm{R} \$ 0,80$.

Além das perdas por trincas e quebra dos ovos, os altos níveis de vibração e choques podem diminuir a qualidade dos ovos férteis. Devido ao agitamento do material interno do ovo tem-se a danificação do albúmen e da qualidade da gema que, consequentemente, provocará mortalidade e máformação embrionária além de reduzir a qualidade dos pintos de corte (Thompson et al., 1986; Altuntaş \& Şekeroğlu, 2008). Devido ao alto nível de vibração e choques da carga os ovos férteis recém-coletados (ovos novos) podem apresentar características e aparência de mais velhos, ou seja, eles poderão ter características semelhantes às dos ovos que permaneceram estocados por 10 dias (Berardinelli et al., 2003).

\section{ConclusõEs}

1. Os piores níveis de vibração e choque da carga de ovos ocorreram no sentido vertical (deslocamento da carga da frente para o fundo) em razão das ultrapassagens, frenagens bruscas e alta velocidade.

2. As estradas de asfalto foram as que provocaram os maiores níveis de vibração e choques, quando comparados aos da estrada de terra.

3. As perdas pré-porteiras nas operações de transporte de ovos ultrapassam $1 \%$, as quais são atribuídas ao número de ovos trincados e quebrados.

\section{Agradecimentos}

Os autores agradecem à Fundação de Amparo à Pesquisa do Estado de São Paulo (FAPESP), pelo apoio financeiro a esta pesquisa e à CAPES, pela concessão da bolsa de estudo.

\section{Literatura Citada}

Altuntaş, E.; Şekeroğlu, A. Effect of egg shape index on mechanical properties of chicken eggs. Journal of Food Engineering, v.85, p.606-612, 2008. 
Bain, M. M.; Dunn, I. C.; Wilson, P. W.; Joseph, N.; Ketelaere, B. de; Baerdemaeker, J.; Waddington, D. Probability of aneggcracking during packing can be predicted using a simple non-destructive acoustic test. British Poultry Science, v.47, p.462-469, 2006.

Bamelis, F. R.; Ketelaere, B. de; Mertens, K.; Kemps, B. J.; Decuypere, E. M.; Baerdemaeker, J. G. de. Measuring the conductance of eggshells using the acoustic resonance technique and optical transmission spectra. Computers and Electronics in Agriculture, v.62, p.35-40, 2008.

Berardinelli, A.; Donati, V.; Giunchi, A.; Guarnieri, A.; Ragni, L. Effects of transport vibrations on quality indices of shell eggs. Biosystems Engineering, v.86, p.495-502, 2003.

Deng, X.; Wang, Q.; Chen, H.; Xie, H. Egg shell crack detection using a wavelet-based support vector machine. Computers and Electronics in Agriculture, v.70, p.135-143, 2010.

Franchini, D. Análise do nível de vibrações verticais no assento de um trator agrícola. Santa Maria: UFSM, 2007. 139p. Dissertação Mestrado

Idah, P. A.; Yisa, M. G.; Ajisegiri, E. S. A.; Okpala, O. Resonance frequency of Nigerian tomato fruit as related to prevention of damage during transportation. Journal of Food Science and Technology, v.46, p.153-155, 2009.

ISO 2631-1.Mechanical vibration and shock - Evaluation of human exposure to whole-body vibration - Part 1: General requeriments. New York: Acoustical Society of America, 31p. 1997.

Lida, I. Ergonomia: Projeto e produção. 2.ed. São Paulo: Edgard Blücher, 420p. 2005.

Mertens, K.; Bamelis, F.; Kemps, B.; Kamers, B.; Verhoelst, E.; Ketelaere, B. de; Bain, M.; Decuypere, E.; Baerdemaeker, J. G. de. Monitoring of eggshell breakage and eggshell strength in different production chains of consumption eggs. Poultry Science, v.85, p.1670-1677, 2006.

Mertens, K.; Ketelaere, B. de; Kamers, B.; Bamelis, F. R.; Kemps, B.J.; Verhoelst, E. M.; Baerdemaeker, J. G. de; Decuypere, E. M. Dirt detection on brown eggs by means of color computer vision. Poultry Science, v.84, p.1653-1659, 2005.
Nazareno, A. C. Ambiência pré-porteira: Avaliação das condições bioclimáticas e das operações pré-eclosão na qualidade de pintos de corte.Piracicaba: ESALQ/USP, 2012. 207p. Tese Doutorado

Pierce, C.D.; Singh, S.P; Burgess, G. A comparison of leafspring with air-cushion trailer suspension in the transport environment. Packaging Technology and Science, v.5, p.11-15, 1992.

Randall, J. M.; Duggan, J. A.; Alami, M. A.; White, R. P. Frequency weightings for the aversion of broiler chickens to horizontal and vertical vibration. Journal of Agricultural Engineering Research, v.68, p.387-397, 1997.

Randall, J.M.; Streader, W. V.; Meehan, A.M. Vibration on poultry transporters. British Poultry Science, v.34, p.635$642,1993$.

SAS Institute. Statistical analysis system: Realease 9.2, (software). Cary, 2010.620p.

Thompson, B.K.; Thompson, R.M.G.; Hamilton, R. M. G. Relationship between laboratory measures of shell strength and breakage of eggs collected at a commercial grading station. Poultry Science, Savoy, v.65, p.1877885, 1986.

Turra, F. Pecuária - Os preços das carnes decolaram tanto aqui como lá fora. Revista Rural, v.14, p.3, 2012.

Walber, M.; Tamagna, A. Avaliação dos níveis de vibração existentes em passageiros de ônibus rodoviários intermunicipais, análise e modificação projetual. Revista Liberato, v.11, p.1-88, 2010.

Wang, J.; Jiang, R.S. Eggshell crack detection by dynamic frequency analysis. European Food Research and Technology, v.221, p.214-220, 2005.

Zhang, L.; Yue , H. Y.; Zhang, H. J.; Xu , L.; Wu, S. G.; Yan, H. J.; Gong, Y. S.; Qi, G. H. Transport stress in broilers: I. Blood metabolism, glycolytic potential, and meat quality. Poultry Science, v.88, p.2033-2041, 2009. 